\title{
Cámaras trampa y huemules: ¿una alternativa de monitoreo?
}

\author{
Camera traps and huemul deer: \\ an alternative for monitoring?
}

Alejandro R. Vila ${ }^{1}$, Gustavo Aprile ${ }^{2}$, Víctor Sotelo ${ }^{3}$, Pablo Sugliano ${ }^{3}$, Carlos Zoratti ${ }^{3}$, Mauricio Berardi ${ }^{4} \&$ Javier Montbrun ${ }^{4}$

El uso de cámaras disparadas en forma remota para fotografiar fauna silvestre se remonta a 1877, pero el método fue poco utilizado hasta la invención de cámaras con sensores automáticos infrarrojos en los años 80 (Kelly et al. 2012). Con el rápido desarrollo comercial de las cámaras trampa y la incorporación de la tecnología digital, en los últimos 20 años se produjo una explosión en su uso como una herramienta para realizar estudios de ecología y conservación. Esto se refleja en un crecimiento anual del $50 \%$ en publicaciones que involucraron el uso de cámaras trampa durante la década pasada (Rowcliffe \& Carbone, 2008; McCallum, 2013). Entre sus aplicaciones se destacan: inventarios de fauna y detección de especies elusivas o amenazadas; estimaciones de abundancia relativa; estimaciones de abundancia, densidad, supervivencia y reclutamiento de especies con individuos que sean identificables; desarrollo de modelos de ocupación; estudios de uso de hábitat, dieta y patrones de actividad (Rovero et al. 2013); y la evaluación de presiones antrópicas como la caza (Di Bitetti et al. 2008).

El huemul (Hippocamelus bisulcus Molina, 1782) es un ciervo de tamaño mediano, endémico los bosques andinopatagónicos y pastizales subandinos, que está considerado como en peligro de extinción (Vila et al. 2010). Sus poblaciones son difíciles de monitorear porque, en general, presentan baja densidad de individuos, están ubicadas en áreas poco accesibles y/o en zonas boscosas que dificultan la observación directa y el conteo de ejemplares (Wittmer et al. 2010). Esto ha motivado que la mayoría de los estudios para evaluar presencia y/o abundancia de la especie estén basados en indicadores indirectos (Povilitis, 1998, 2002; Serret \& Borghiani, 1998). Por esta razón, en este trabajo pusimos a prueba el potencial de las cámaras trampa para monitorear poblaciones de huemul en tres áreas protegidas de la República Argentina.

El estudio se desarrolló, entre 2007 y 2011 , en nueve sitios piloto (una cámara por sitio) ubicados en el Parque Nacional Los Alerces (2 sitios), provincia del Chubut, la Reserva Privada Ea. Los Huemules (6) y el Parque Nacional Los Glaciares (1), provincia de Santa Cruz, Argentina. Se utilizaron cámaras DeerCam DC-200 con película y Cuddeback Capture y Expert digitales. Las mismas fueron ubicadas sobre sendas o sitios con presencia previa de signos y/o con hábitat que se consideró apropiado para la especie (Vila, 2005; Díaz et al. 2013). Las cámaras se mantuvieron activas en promedio 327 días por sitio (rango= 39632).

Se obtuvieron un total de 105 fotografías de huemules. En algunos casos, la cámara tomó

Wildlife Conservation Society, Amenabar 1595, 2do. 19 (1426), Buenos Aires, Argentina. $\sum$ avila@wcs.org

2 ACEN, Serrano 1779 (1663) San Miguel, Buenos Aires, Argentina .

3 Administración de Parques Nacionales, Parque Nacional Los Glaciares, Argentina.

4 Administración de Parques Nacionales, Parque Nacional Los Alerces, Argentina. 
más de una foto (2 a 12) del mismo individuo con minutos de diferencia o imágenes en las que se repite el mismo ejemplar, pero con días de diferencia entre las distintas tomas. El Cerro Polo fue el sitio donde se lograron más fotos (47), seguido por la Laguna Azul (32). En el río y la loma del Diablo las cámaras no tomaron fotos de la especie. Se fotografiaron individuos de todas las categorías de edad y sexo (Figs. 1 y 2), principalmente machos y hembras adultas, 36 y $39 \%$ del total de las fotos respectivamente. Se lograron fotos durante todas las estaciones del año (Figs. 2 y 3), con mayor número de registros durante el invierno $(24 \%$ del total de fotos) y la primavera (42\%); y en todos los momentos del día, incluso durante la noche (Fig. 2). La tasa media de capturas (fotos/100 días) fue 15 (rango=0,28 en Leonera y 34 en Punta Mattos), sin incluir en el análisis los sitios en donde no se tomaron fotos (Tabla 1). A partir del sexo de los individuos, los patrones de las astas de los machos, tamaño, coloración del pelo o marcas en el pelaje, se lograron individualizar ejemplares y establecer números mínimos de individuos fotografiados, 19 en total y entre 1 a 6 por sitio (Tabla 1). Asimismo, las cámaras también permitieron detectar la presencia de algunas amenazas potenciales (Fig. 2), como vacunos (Bos taurus) y caballos (Equus caballus) -competencia o posible transmisión de enfermedades-, pumas (Puma concolor) y zorros culpeos (Dusicyon culpaeus) -depredación- y personas que podrían provocar disturbios en algunos de los sitios de muestreo. Finalmente, las cámaras digitales tuvieron un mejor rendimiento en cuanto al consumo de baterías, alcanzando hasta un máximo de 72 a 102 días de funcionamiento continuo.

Los resultados de estudio preliminar muestran el potencial de las cámaras trampa para monitorear poblaciones de huemules. En particular, para identificar la presencia de la especie y la ocurrencia de reproducción (presencia de crías y juveniles), realizar estimaciones de abundancia relativa (frecuencias de captura), evaluar patrones diarios y estacionales de actividad (las fotos aportan la fecha y hora de la toma), uso de hábitat y amenazas. Si bien la identificación de algunos ejemplares fue posible, resultó compleja como para utilizar métodos de captura y recaptura comúnmente empleados en carnivoros (Karanth \& Nichols, 1998; Silver et al. 2004; Kelly et al. 2008). A pesar de ello, sería factible obtener índices de abundancia relativa (capturas/días trampa) como los presentados en nuestro estudio y comparar sitios a través de diseños de muestreo

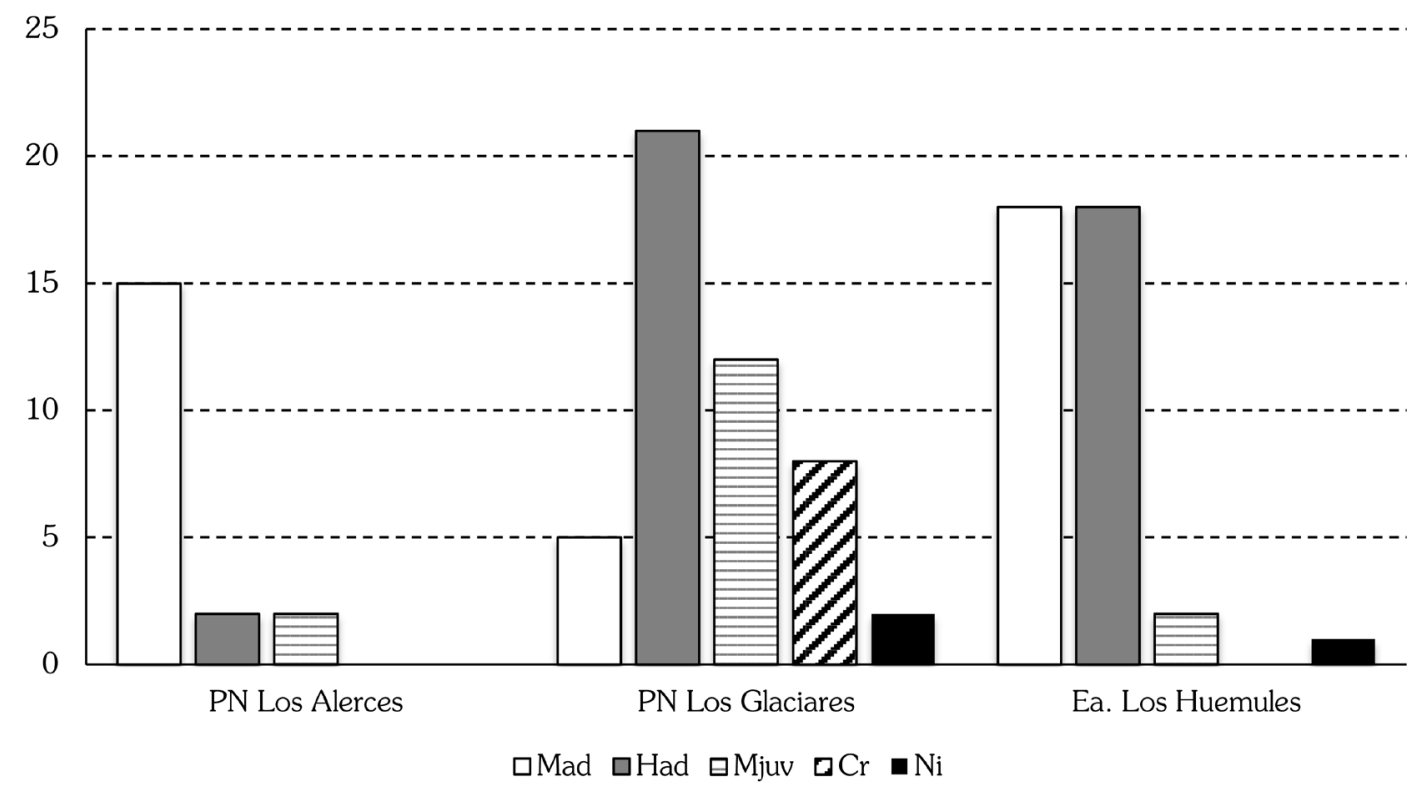

Fig. 1. Número de fotografías obtenidas con cámaras trampas por categoría de edad y sexo para cada área protegida (PN= Parque Nacional). Mad; Macho Adulto, Had: Hembra Adulta, Mjuv: Macho Juvenil, Cr: Cría y Ni: No Identificado. 

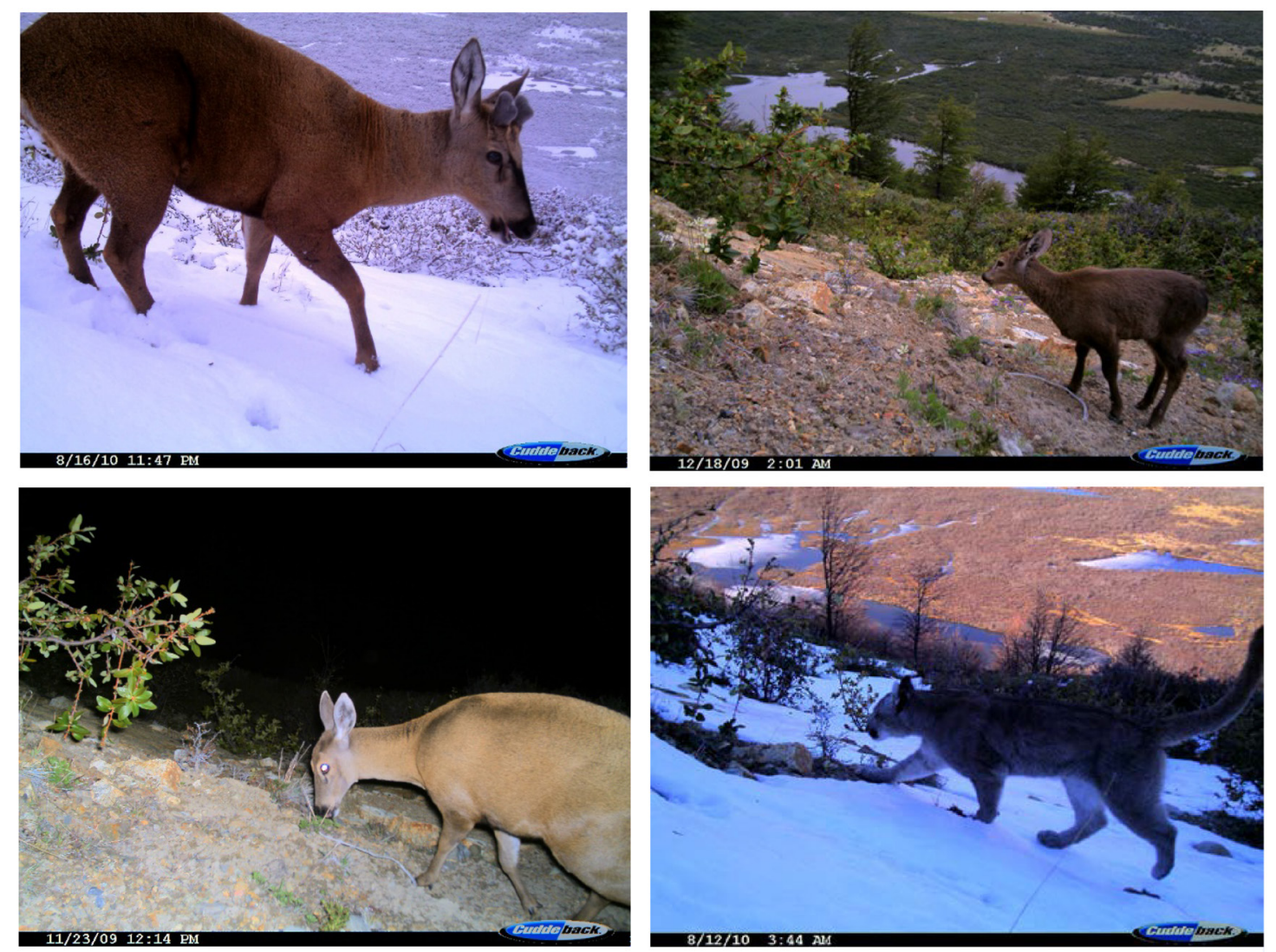

Fig. 2. Ejemplos de fotografías tomadas con las cámaras trampa en el Parque Nacional Los Glaciares, Argentina. Arriba izquierda: macho adulto con astas en felpa en invierno, y derecha: cría de la temporada a fines de la primavera. Abajo izquierda: hembra adulta alimentándose durante la noche, y derecha: presencia de puma (depredador del huemul).

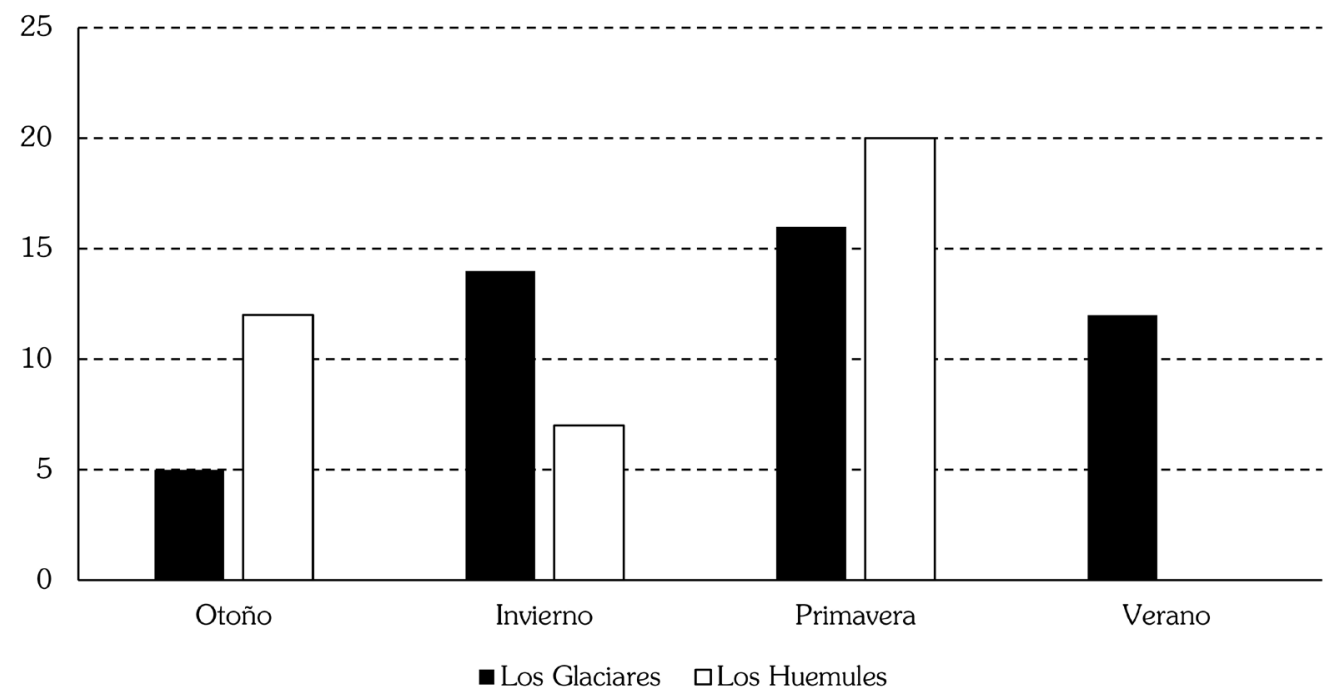

Fig. 3. Número de fotografías obtenidas estacionalmente con cámaras trampa para el Parque Nacional Los Glaciares y la Reserva Privada Los Huemules (Argentina), en los cuales se mantuvo un monitoreo más intensivo a lo largo del año. 
Tabla 1. Resumen de los resultados obtenidos para el huemul con cámaras trampas en tres áreas protegidas de Argentina ( $\mathrm{PN}=$ Parque Nacional), indicando sitios, días de muestreo, número de fotos obtenidas, tasa de fotos cada 100 días y número mínimo de individuos estimados.

\begin{tabular}{|c|c|c|c|c|c|}
\hline Área Protegida & Sitios & Días & Fotos & $\begin{array}{c}\text { Tasa } \\
\text { (x } 100 \mathrm{~d})\end{array}$ & $\begin{array}{l}\text { \# Mín. } \\
\text { individuos }\end{array}$ \\
\hline \multirow{4}{*}{ PN Los Alerces } & Quebrada del León & 215 & 3 & 1,40 & 2 \\
\hline & $42^{\circ} 48^{\circ} 06^{\prime \prime} \mathrm{S} / 71^{\circ} 40^{-1} 17^{\prime \prime}(650 \mathrm{msnm})$ & & & & \\
\hline & Pta. Mattos & 47 & 16 & 34,04 & 3 \\
\hline & $42^{\circ} 48^{\circ} 51^{\prime \prime} \mathrm{S} / 71^{\circ} 42^{\circ} 48^{\prime \prime}$ (591 msnm) & & & & \\
\hline \multirow{2}{*}{ PN Los Glaciares } & Cerro Polo & 632 & 47 & 7,44 & 6 \\
\hline & $49^{\circ} 17^{\prime} 18^{\prime \prime} \mathrm{S} / 72^{\circ} 54^{\prime} 23^{\prime \prime} \mathrm{O}$ (571 msnm) & & & & \\
\hline \multirow{12}{*}{ Los Huemules } & Laguna Verde & 340 & 4 & 1,18 & 2 \\
\hline & $49^{\circ} 12^{\prime} 29^{\prime \prime} \mathrm{S} / 72^{\circ} 58^{\prime} 18^{\prime \prime O}$ (566 msnm) & & & & \\
\hline & Río del Diablo & 140 & 0 & 0,00 & 0 \\
\hline & $49^{\circ} 12^{-2} 27^{\prime \prime} \mathrm{S} / 72^{\circ} 58^{-17}$ ”O (576 msnm) & & & & \\
\hline & Laguna Azul & 590 & 32 & 5,42 & 4 \\
\hline & $49^{\circ} 13^{\prime} 09^{\prime \prime} \mathrm{S} / 72^{\circ} 59^{\prime} 04^{\prime \prime} \mathrm{O}$ (610 msnm) & & & & \\
\hline & Acarreo Loma del Diablo & 39 & 0 & 0,00 & 0 \\
\hline & $49^{\circ} 11^{\circ} 08^{\prime \prime S} / 72^{\circ} 57^{\circ} 26^{\prime \prime O}(650 \mathrm{msnm})$ & & & & \\
\hline & Leonera 2 & 362 & 1 & 0,28 & 1 \\
\hline & $49^{\circ} 13^{\prime} 42^{\prime \prime} \mathrm{S} / 72^{\circ} 58^{\prime} 8^{\prime \prime} \mathrm{O}$ (476 msnm) & & & & \\
\hline & Zona Intangible & 578 & 2 & 0,35 & 1 \\
\hline & 49¹2'58”S / 7259’7”O (604 msnm) & & & & \\
\hline
\end{tabular}

similares a los utilizados en carnívoros (Silver, 2004) y otros mamíferos medianos y grandes (Tobler et al. 2008).

Estudios recientes en otras poblaciones de huemules también han puesto a prueba el potencial de las cámaras trampa y demostrado su efectividad en Chile. Por ejemplo, han sido utilizadas en la Reserva Nacional Nuble para determinar la presencia estacional de la especie y sus depredadores (Hinojosa et al. 2013; Bonacic et al. 2013), números mínimos, patrones de actividad diaria y la asociación con algunas variables de hábitat (Bonacic et al. 2013). El éxito de captura reportado por Bonacic et al. (2013), 0,43 huemules por 100 días de cámaras trampa, resultó comparable al registrado en los sitios con menor tasa de captura de nuestro estudio. Por su parte, López (2015 y 2016) utilizó cámaras trampa en forma combinada con signos de presencia de la especie para monitorear una población del Área de Alto Valor de la Región del Biobío; mientras que Sandvig et al. (2016) identificaron, por medio de cámaras trampa, cuáles son las variables ambientales que explican la presencia del huemul en un área con plantaciones de pino ponderosa (Pinus ponderosa Douglas, 1826) y bosque nativo de la región de Aysén, utilizando modelos de ocupación.

Considerando el rendimiento y los costos asociados, las cámaras digitales fueron superiores a las de rollo en nuestro estudio, pues consumieron menos batería y las fotos pudieron ser descargadas in-situ. Incluso, las cámaras con paneles solares podrían mejorar aún más la performance, pues están dando buenos resultados en proyectos de ciervos colorados (Cervus elaphus) y gatos huiña (Leopardus guigna) en áreas andino-patagónicas (N. Ferreyra y M. Monteverde, com. pers.).

En síntesis, además de los beneficios enumerados en este estudio en cuanto al uso 
potencial de cámaras para estudiar especies elusivas $y$ de baja densidad como el huemul, se puede mencionar que es posible monitorear su presencia, actividad y categorías de edad y sexo en sitios de estudio a lo largo de todo el año y durante todo el día, como así también asociaciones de hábitat y presencia de potenciales amenazas, incluso con un bajo número de cámaras que pueden rotarse entre sitios. Sin embargo, para desarrollar estudios tendientes a determinar estimaciones de abundancia y patrones de uso, selección de hábitat y actividad se requiere de un número mayor de cámaras $y$, por lo tanto, un mayor esfuerzo de campo y un importante costo de inversión inicial en equipos. Otras desventajas a considerar son el potencial robo de las cámaras o actos de vandalismo sobre las mismas, y el uso y disposición final de baterías con sus efectos ambientales asociados. Si bien la inversión inicial en comprar las cámaras es alta, su vida útil es prolongada y pueden ser compartidas, rotadas $\mathrm{O}$ utilizadas colaborativamente entre diferentes equipos de trabajo para potenciar tanto el esfuerzo de muestreo (más cámaras y más sitios bajo monitoreo) como reducir costos para cada proyecto individual. Asimismo, en términos de horas/persona y costos en terreno para relevar presencia por medio de observación directa de signos o ejemplares, las cámaras trampa permiten reducir estos costos considerablemente.

\section{AGRADECIMIENTOS}

Este trabajo se realizó con autorización de la Administración de Parques Nacionales y el Consejo Agrario de la Provincia de Santa Cruz. Las tareas de campo se implementaron gracias al apoyo de N. Alvarez, M. Cravea, P. Díaz, G. Fanelli, M. Gray, A. Randazzo, F. Reese, C. Sans, A. Serret y C. Sugliano. Las cámaras utilizadas fueron gentilmente facilitadas por Idea Wild. Asimismo, se agradecen los comentarios y sugerencias de dos revisores anónimos para mejorar este trabajo.

\section{LITERATURA CITADA}

Bonacic, C., Ohrens, O., Forero, L. M., Petitpas, R. Guarda, N., \& Zumaeta, C. (2013). Informe Proyecto Huemul Nuble. Santiago, Chile: Facultad de Agronomía e Ingeniería Forestal,
Pontificia Universidad Católica de Chile.

Díaz, P., Marqués, B. I., \& Vila, A. R. (2013). Seasonal habitat use and selection of the endangered huemul deer (Hippocamelus bisulcus) in Patagonian Andes. Mammalia, 77, 371-380.

Di Bitetti, M. S., Paviolo, A., Ferrari, C. A., De Angelo, C., \& Di Blanco, Y. (2008). Differential responses to hunting in two sympatric species of brocket deer (Mazama americana and M. nana). Biotropica, 40(5), 636-645.

Hinojosa, A., Ramírez, E., \& Peralta, A. (2013). Uso de cámaras trampa para monitoreo de fauna. Chile Forestal, 365, 42-45.

Karanth, K., \& Nichols, J. (1998). Estimation of tiger densities in India using photographic captures and recaptures. Ecology, 79, 2852-2862.

Kelly, M. J., Noss, A. J., Di Bitetti, M. S., Maffei, L., Arispe, R. L., Paviolo, A., De Angelo, C., \& Di Blanco, Y. E. (2008). Estimating puma densities from camera trapping across three study sites: Bolivia, Argentina, and Belize. Journal of Mammalogy, 89(2), 408-418.

Kelly, M. J., Betsch, J., Wultsch, C., Mesa, B., \& Mills, L. S. (2012). Noninvasive sampling for carnivores. En L. Boitani, \& R. A. Powell (Eds.), Carnivore ecology and conservation: a handbook of techniques (pp. 47-69). New York: Oxford University Press.

López, R. (2015). Monitoreo del huemul (Hippocamelus bisulcus) en el área de alto valor de conservación (AAVC) Rucamanqui. Concepción, Chile: Forestal Mininco.

López, R. (2016). Plan de monitoreo del huemul (Hippocamelus bisulcus) en el área de alto valor de conservación (AAVC) Huemules de Nuble. Concepción, Chile: Arauco.

McCallum J., (2013). Changing use of camera traps in mammalian field research: habitats, taxa and study types. Mammal Review, 43(3), 196-206.

Povilitis, A. (1998). Characteristics and conservation of a fragmented population of huemul Hippocamelus bisulcus in central Chile. Biological Conservation, 86, 97104. 
Povilitis, A. (2002). El estado actual del huemul (Hippocamelus bisulcus) en Chile Central. Gayana, 66, 59-68.

Rovero, F., Zimmermann, F., Berzi, D., \& Meek, P. (2013). Which camera trap type and how many do I need? A review of camera features and study designs for a range of wildlife research applications. Italian Journal of Mammalogy, 24(2), 148-156.

Rowcliffe, J. M., \& Carbone, C. (2008). Surveys using camera traps: are we looking to a brighter future? Animal Conservation, 11(3), 185-186.

Sandvig, E. M., Espinaze, M., Marin-Vial, P., \& Corti, P. (2016). Assessing productive lands as viable habitat for huemul in patagonia. Journal of Wildlife Management, 80(3), 573-578.

Serret, A., \& Borghiani, F. (1998). Situación comparada del estado de conservación del huemul -entre 1988 y 1998-en los Lagos Nansen y Azara, P.N. Perito Moreno, Prov. de Santa Cruz. Buenos Aires, Argentina: Boletín Técnico FVSA 45.

Silver, S., Ostro, L., Marsh, L., Maffei, L., Noss, A., Kelly, M., Wallace, R.,...Ayala, G. (2004). The use of camera traps for estimating jaguar Panthera onca abundance and density using capture/recapture analysis. Oryx, 38, 148-
154.

Silver, S. (2004). Estimando la abundancia de jaguares mediante trampas-cámara. New York, US: Programa para la Conservación del Jaguar, WCS.

Tobler, M. W., Carrillo-Percastegui, S. E., Leite Pitman, R., Mares, R., \& Powell, G. (2008). An evaluation of camera traps for inventorying large-and medium-sized terrestrial rainforest mammals. Animal Conservation, 11(3), 169-178.

Vila, A. R. (2005). Línea de base para el huemul (Hippocamelus bisulcus) en la Estancia Los Huemules. Caleta Olivia, Argentina: UNPA-CPSA.

Vila, A. R., Saucedo Galvez, C. E., Aldridge, D., Ramilo, E., \& Corti González, P. (2010). South Andean Huemul (Hippocamelus bisulcus, Molina 1782). En J. M. Barbanti Duarte \& S. González (Eds.), Neotropical Cervidology: Biology and Medicine of Latin American Deer (pp. 89-100). San Pablo, Brasil: FUNEP-IUCN.

Wittmer H. U., Corti, P., Saucedo, C., \& Galáz, J. L. (2010). Learning to count: adapting population monitoring for endangered huemul deer Hippocamelus bisulcus to meet conservation objectives. Oryx, 44, 516-522. 